\title{
Association of extracellular matrix metalloproteinase inducer in endometrial carcinoma with patient outcomes and clinicopathogenesis using monoclonal antibody $12 \mathrm{C3}$
}

\author{
KAZU UEDA ${ }^{2}$, KYOSUKE YAMADA ${ }^{2}$, MITSUYOSHI URASHIMA ${ }^{1}$, YOSHIO ISHIBASHI ${ }^{3}$, \\ MISAKO SHIRAI ${ }^{4}$, TAKASHI NIKAIDO ${ }^{4}$, HIROYUKI TAKAHASHI ${ }^{4}$, AIKOU OKAMOTO $^{2}$, \\ MISATO SAITO $^{2}$, MAKOTO YASUDA ${ }^{2}$, KIYOSHI OHKAWA ${ }^{5}$ and TADAO TANAKA ${ }^{2}$ \\ ${ }^{1}$ Division of Clinical Research and Development; Departments of ${ }^{2}$ Obstetrics and Gynecology, ${ }^{3}$ Surgery, ${ }^{4}$ Pathology \\ and ${ }^{5}$ Biochemistry, Jikei University School of Medicine, 3-25-8 Nishi-shinbashi, Minato-ku, Tokyo 105-8461, Japan
}

Received October 6, 2006; Accepted December 22, 2006

\begin{abstract}
Extracellular matrix metalloproteinase inducer (EMMPRIN) is a member of the immunoglobulin superfamily of adhesion molecules and has a role in the activation of several matrix metalloproteinases (MMPs). We evaluated whether EMMPRIN expression is related to tumor progression and patient outcome in human endometrial carcinoma. Paraffinembedded surgical tissue samples from 112 patients with endometrial carcinoma were stained with anti-EMMPRIN antibody (monoclonal antibody 12C3:MoAb 12C3) for immunohistochemical analysis. EMMPRIN protein was expressed in cancerous lesions with the incidence of $97.3 \%$ (109 of 112 cases), but not in normal lesions. The scores determined by the combination of intensity and pattern of EMMPRIN staining in cancer cells correlated significantly with various histopathological risk factors: advanced stage, $\mathrm{P}=0.001$; poorly differentiated carcinoma, $\mathrm{P}<0.001$; lymph node metastasis, $\mathrm{P}=0.002$; and lymphatic vessel infiltration, $\mathrm{P}=0.027$. More importantly, recurrence-free survival was shortened in patients with higher EMMPRIN scores (HR, 3.08; 95\% CI, 1.32-7.19; $\mathrm{P}=0.01)$. These results suggest that measurement of EMMPRIN expression with simple immunohistochemical staining may enhance the understanding of the pathophysiology of endometrial carcinoma.
\end{abstract}

\section{Introduction}

Matrix metalloproteinases (MMPs) are endopeptidases that play critical roles in promoting tumor disease progression,

Correspondence to: Dr T. Tanaka, Department of Obstetrics and Gynecology, Jikei University School of Medicine, 3-25-8 Nishishinbashi, Minato-ku, Tokyo 105-8461, Japan

E-mail: tanaka3520@jikei.ac.jp

Key words: EMMPRIN, endometrial carcinoma, monoclonal antibody, recurrence-free survival including tumor angiogenesis. In many solid tumors, MMP expression could be attributed to tumor stromal cells and is partially regulated by tumor-stroma interactions by means of tumor cell-associated extracellular matrix metalloproteinase inducer (EMMPRIN) (1). The roles of EMMPRIN and MMPs in tumor invasiveness have been confirmed immunohistochemically in several types of cancer cells (2-4). Moreover, research on EMMPRIN in malignant disease has recently attracted attention, and the expression of EMMPRIN has been reported to correlate with clinical prognosis of patients with breast carcinoma $(5,6)$, ovarian carcinoma (7) and other types of cancer (8-11).

The prognosis for endometrial carcinoma patients with early clinical stage and well-differentiated carcinoma is generally satisfactory, but advanced stage and/or poorly differentiated carcinoma is an aggressive tumor with a poor prognosis (12-14). It would be beneficial to elucidate the pathophysiology of endometrial carcinoma concerning tumor invasiveness and differentiation.

We have established a murine monoclonal antibody (MoAb) 12C3 (15) that specifically binds to EMMPRIN protein (8). In the current study, EMMPRIN protein-expression patterns in endometrial carcinoma were examined immunohistochemically using MoAb 12C3 to determine their relation to clinicopathologic findings and recurrence-free survival.

\section{Materials and methods}

Tumor specimens. The Jikei University School of Medicine Ethics Review Committee approved the study protocol. A total of 112 endometrial carcinoma operative specimens were retrospectively obtained at the Jikei University Hospital (Tokyo, Japan) between January 1998 and March 2003. Tumors were histologically classified according to the WHO international system and the clinical cancer staging and the histological grade were defined according to the International Federation of Gynecology and Obstetrics (Table I). All of the 112 cases underwent hysterectomy, bilateral salpingooophorectomy and lymphadenectomy (or pelvic lymph node sampling). No cases received chemotherapy, radiotherapy or hormone therapy before they underwent operation. 
Table I. Patient characteristics.

\begin{tabular}{lc}
\hline & $\mathrm{n}=112$ \\
\hline Age (mean \pm SD, years) & $55.3 \pm 11.7$ \\
FIGO stage $^{\mathrm{a}}$ & \\
I & 68 \\
II & 18 \\
III & 21 \\
IV & 5 \\
Histological type & \\
Endometrioid & 101 \\
Serous & 4 \\
Mucinous & 1 \\
Others & 6 \\
\hline
\end{tabular}

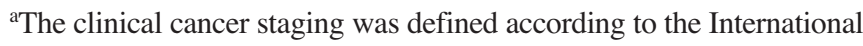
Federation of Gynecology and Obstetrics.

Inohistochemical analysis. For the immunohistochemical study, formalin-fixed paraffin-embedded sections were used. Immunostaining was performed using the labeled streptavidinbiotin peroxidase complex method with the Ventana autoimmunostaining system (Ventana Japan, Yokohama, Japan). A murine MoAb 12C3 against EMMPRIN protein was established as described (8). The antigen retrieval procedure was performed with a microwave oven in Dako antigen retrieval solution for $10 \mathrm{~min}$ at $95^{\circ} \mathrm{C}$ to efficiently stain the sample. The sections (Dako Cytomation, Glostrup, Denmark) were developed with 3,3'-diaminobenzidine with $0.3 \% \mathrm{H}_{2} \mathrm{O}_{2}$ and counterstained with hematoxylin. As a negative control, pre-immune mouse serum diluted 100 -fold with $1 \%$ bovine serum albumin (BSA; Sigma, St. Louis, MO) in $20 \mathrm{mM}$ Tris$\mathrm{HCl}, \mathrm{pH} 7.6,0.5 \mathrm{M} \mathrm{NaCl}$ (TBS) was used instead of MoAb.

Results of staining for EMMPRIN in cancerous lesions were evaluated using the following scoring system. The intensity of staining was classified into negative (0), weak (1), strong (2) or very strong (3), and the staining patterns were classified into negative (0), sporadic (1), focal (2) or diffuse (3), respectively and the total sum was evaluated. The examiners were blinded to patient clinicopathologic information when assigning staining intensity and patterns. Four investigators (K.U., K.Y., H.T. and M.U.) evaluated the staining results independently, after which discordant evaluations were adjusted by connected microscopes and scored finally.

Statistical analysis. $\chi^{2}$ tests were used to evaluate the relationship between immunohistochemical scores and several clinicopathologic parameters. Survival curves of the patients were compared using the Kaplan-Meier method and analyzed by the log-rank test. Cox proportional hazard models were fitted for univariate and multivariate analysis. All these analyses were performed using STATA 8.0 (STATA Corp., College Station, TX).
A

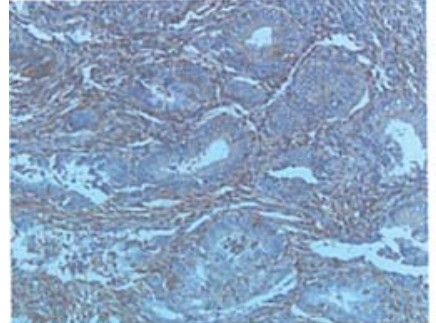

(a) negative

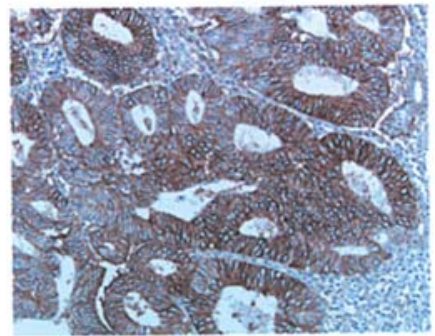

(c) strong

B

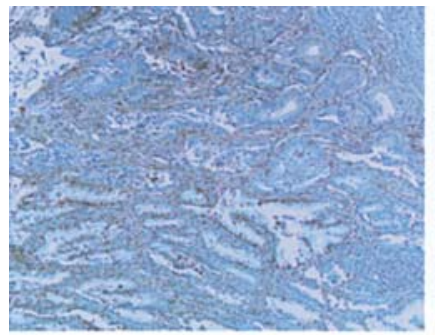

(a)negative

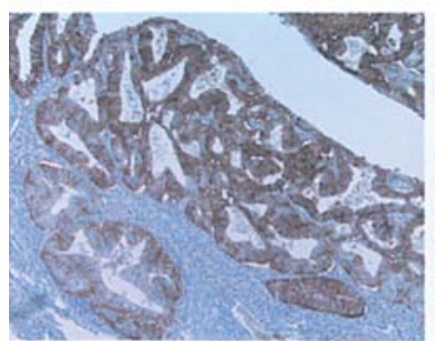

(c) focal

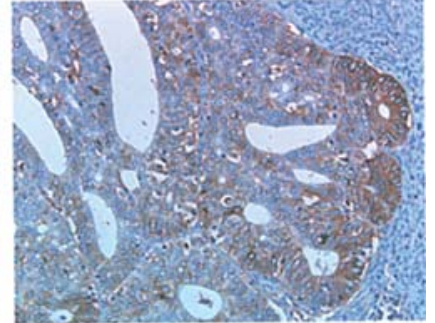

(b) weak

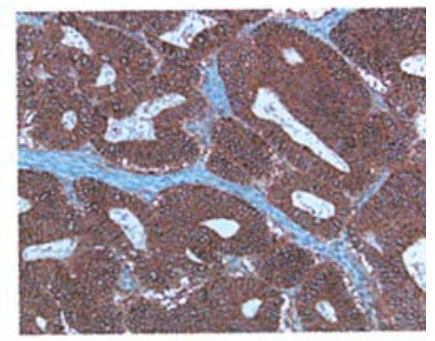

(d) very strong

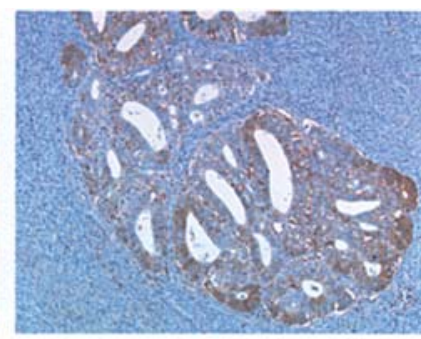

(b) sporadic

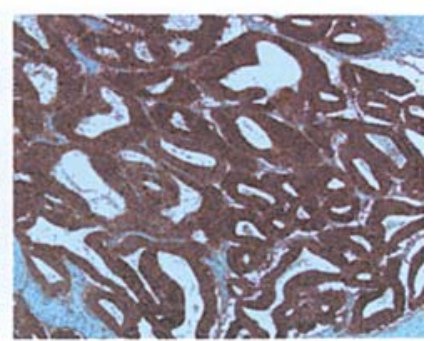

(d) diffuse
Figure 1. Immunohistochemical analysis and typical findings of EMMPRIN staining (intensity and pattern) in endometrial carcinoma using MoAb 12C3. EMMPRIN protein was expressed in cancerous lesions but not in normal lesions including the stromal cells and myometrium. (A) The intensity of staining was classified into negative (a), weak (b), strong (c), or very strong (d), respectively (magnification x200). (B) The staining patterns were classified into negative (a), sporadic (b), focal (c), or diffuse (d), respectively (magnification $\mathrm{x} 100)$.

\section{Results}

Protein expression of EMMPRIN. MAb 12C3 reacted in 109 of 112 cases $(97.3 \%)$ of endometrial carcinoma. EMMPRIN protein was expressed in cancerous lesions but not in normal lesions including the stromal cells and myometrium. Typical findings of EMMPRIN immunohistochemical staining (intensity and pattern) of paraffin-embedded specimens are 


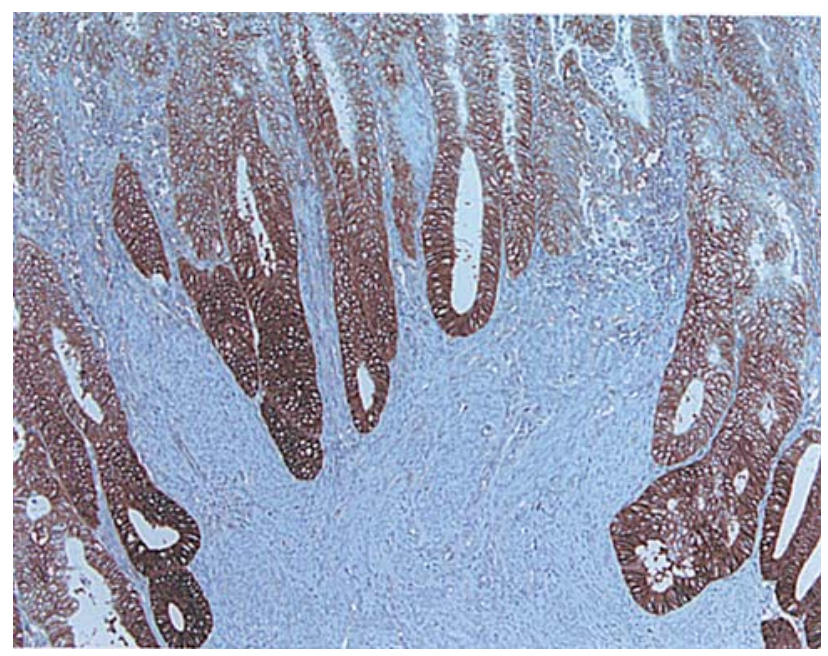

Figure 2. Immunohistochemical staining of endometrioid adenocarcinoma (Stage IIb, well-differentiated adenocarcinoma). EMMPRIN staining intensity was strong in deep cancer lesions with comparative examinations of serial tissue sections (magnification x100).

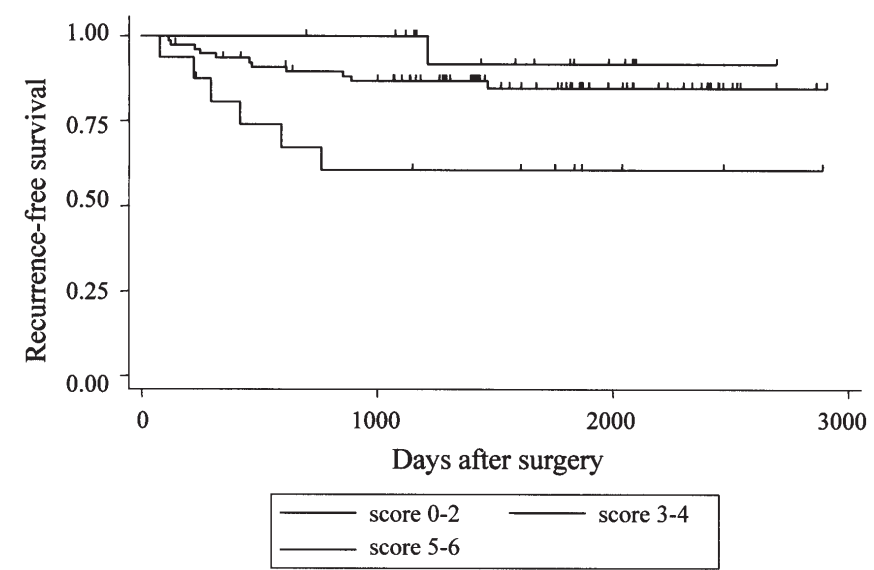

Figure 3. Kaplan-Meier survival curves by scores of EMMPRIN staining. Patients were grouped into three categories on expression of EMMPRIN: i) score $0-2$; ii) score 3-4; iii) score 5-6. Statistical differences were analyzed with log-rank test $(\mathrm{p}=0.0153)$.

demonstrated in Fig. 1. In some cases, the staining intensity tended to be strong in deep cancerous lesions compared to shallow lesions (Fig. 2).

Association between clinicopathogenesis and the scoring system. The association between clinicopathogenesis and immunohistochemical scores were evaluated (Table II). The scores reflecting the intensity and the pattern of EMMPRIN staining were significantly higher in advanced stage $(\mathrm{P}=0.001)$, poorly differentiated carcinoma $(\mathrm{P}<0.001)$, lymph node metastasis $(\mathrm{P}=0.002)$, lymphatic vessel infiltration $(\mathrm{P}=0.027)$, the pathological high-risk group $(\mathrm{P}<0.001)$ and patients with recurrence $(\mathrm{P}=0.03)$, respectively.

Survival analyses. Kaplan-Meier analysis on the subgroups of the scores $(0-2,3-4,5-6)$ confirmed the prognostic impact in endometrial carcinoma (log-rank test, $\mathrm{P}=0.0153$ ) (Fig. 3).
Table II. Clinicopathological characteristics of patients by scores of EMMPRIN staining.

\begin{tabular}{|c|c|c|c|c|}
\hline & $\begin{array}{l}\text { Score } 0-2 \\
(\%)\end{array}$ & $\begin{array}{l}\text { Score } 3-4 \\
(\%)\end{array}$ & $\begin{array}{l}\text { Score 5-6 } \\
(\%)\end{array}$ & P-value \\
\hline Stage & & & & 0.001 \\
\hline I & $15(22)$ & $50(74)$ & 3 (4) & \\
\hline II & 1 (6) & $14(78)$ & $3(17)$ & \\
\hline III & $1 \quad(5)$ & $12(57)$ & $8(38)$ & \\
\hline IV & $0 \quad(0)$ & $3(60)$ & $2(40)$ & \\
\hline Grade & & & & $<0.001$ \\
\hline 1 & $16(24)$ & $46(70)$ & $4(6)$ & \\
\hline 2 & 1 (3) & $25(81)$ & $5(16)$ & \\
\hline 3 & $0 \quad(0)$ & $6(46)$ & $7(54)$ & \\
\hline $\mathrm{pN}^{\mathrm{a}}$ & & & & 0.002 \\
\hline 0 & $14(17)$ & $62(76)$ & $6 \quad(7)$ & \\
\hline 1 & 1 (7) & $8(53)$ & $6(40)$ & \\
\hline $\begin{array}{l}\text { Lymphatic vessel } \\
\text { infiltration }\end{array}$ & & & & 0.027 \\
\hline$(-)$ & $14(23)$ & $43(70)$ & $4 \quad(7)$ & \\
\hline$(1+)$ & 1 (4) & $17(68)$ & $7(28)$ & \\
\hline$(2+)$ & $0 \quad(0)$ & $8(73)$ & $3(27)$ & \\
\hline$(3+)$ & $0 \quad(0)$ & $4(80)$ & $1(20)$ & \\
\hline Risk $^{b}$ & & & & $<0.001$ \\
\hline Low & $16(24)$ & $47(70)$ & $4(6)$ & \\
\hline High & 1 (2) & $32(71)$ & $12(27)$ & \\
\hline Recurrence & & & & 0.03 \\
\hline$(-)$ & $16(17)$ & $68(72)$ & $10(11)$ & \\
\hline$(+)$ & 1 (6) & $11(61)$ & $6(33)$ & \\
\hline
\end{tabular}

${ }^{a} 0$, no lymph node metastasis pathologically; 1 , lymph node metastasis. bow-risk patients: no deep myometrial invasion, no uterine cervical invasion and well-differentiated adenocarcinoma; high-risk patients, over stage Ic and/or poorly differentiated carcinoma.

Cox hazard regression analyses were applied to determine the clinicopathological factors associated with recurrencefree survival using a univariate and multivariate manner (Table III). Univariate Cox regression analyses revealed that advanced stage, poorly differentiated carcinoma, lymph node metastasis, lymphatic vessel infiltration, and a high score group of immunohistochemical scoring were significant predictors of recurrence-free survival. On the other hand, multivariate Cox regression analysis showed advanced stage \{hazard ratio, 1.16 [95\% confidence interval (CI), 1.08-1.25] \} and poorly differentiated carcinoma [hazard ratio, $2.35(95 \%$ CI, 1.00-2)] were the only significant poor prognostic factors.

\section{Discussion}

In this study, we determined protein expression of EMMPRIN in cancer cells with immunohistochemical staining, and 
Table III. Univariable and multivariable analyses of factors associated with recurrence-free survival.

\begin{tabular}{|c|c|c|c|c|}
\hline & $\begin{array}{c}\text { Univariable analysis } \\
\text { HR }(95 \% \mathrm{CI})\end{array}$ & P-value & $\begin{array}{c}\text { Multivariable analysis } \\
\text { HR }(95 \% \mathrm{CI})\end{array}$ & P-value \\
\hline Score & $3.08(1.32-7.19)$ & 0.01 & $0.53(0.06-4.45)$ & 0.557 \\
\hline Stage & $1.14(1.08-1.19)$ & $<0.001$ & $1.16(1.08-1.25)$ & $<0.001$ \\
\hline Grade & $3.63(1.98-6.66)$ & $<0.001$ & $2.35(1.00-5.50)$ & 0.049 \\
\hline Risk & $14.67(3.36-63.93)$ & $<0.001$ & $0.03(0.0001-6.54)$ & 0.199 \\
\hline $\mathrm{pN}$ & $15.26(5.18-44.95)$ & $<0.001$ & $0.19(0.01-5.28)$ & 0.326 \\
\hline $\mathrm{pM}^{\mathrm{a}}$ & $7.82(2.22-27)$. & 0.001 & $0.08(0.002-3.13)$ & 0.175 \\
\hline Positive ascites ${ }^{\mathrm{b}}$ & $5.53(2.14-14.32)$ & $<0.001$ & $1.14(0.22-5.83)$ & 0.871 \\
\hline Lymphatic vessel infiltration & $4.52(2.66-7.69)$ & $<0.001$ & $2.32(0.81-6.63)$ & 0.117 \\
\hline
\end{tabular}

a Distant metastasis. ${ }^{\mathrm{b}} \mathrm{Cancer}$ cells in ascites or peritoneal washings.

investigated the association of this protein expression with clinicopathologic findings and recurrence-free survival in 112 patients with endometrial carcinoma. EMMPRIN protein was detected in cancerous lesions but not in normal lesions including the stromal cells and myometrium. Generally, the prognosis of low-risk patients with no deep myometrial invasion, no uterine cervical invasion and well-differentiated adenocarcinoma is satisfactory (16-18). EMMPRIN expression was confirmed not only in high-risk patients with higher stage (over stage Ic) and/or poorly differentiated carcinoma but also in lower risk patients. However, the scores reflecting the intensity and the pattern of EMMPRIN staining in cancer cells were significantly higher in high-risk patients, especially those with advanced stage, poorly differentiated carcinoma, lymph node metastasis, and lymphatic vessel infiltration. The staining scores were associated with recurrence-free survival and seemed to parallel clinical stage. Among stage I cases, although the staining scores were not associated with depth of myometrial invasion statistically, it was notable that the staining intensity was strong in deep cancerous lesions with comparative examinations of serial tissue sections. These results suggested that measurement of EMMPRIN expression with simple immunohistochemical staining might further enhance the understanding of the pathophysiology of endometrial carcinoma.

In clinical treatment, it is important to accurately diagnose surgical staging of endometrial carcinoma by performing systematic lymphadenectomy in addition to hysterectomy, and bilateral salpingo-oophorectomy for decisions concerning adjuvant chemotherapy. However, it is still controversial whether systematic lymphadenectomy can be omitted from remedies for low-risk patients with no deep myometrial invasion and well-differentiated adenocarcinoma. Usually pre-operative evaluation of endometrial carcinoma is mainly performed by D\&C (dilation and curettage) and imaging including ultrasonography and MRI (magnetic resonance imaging), but there is a limit to this procedure in terms of identification of low-risk cases. We were able to validate the clinical importance of EMMPRIN expression retrospectively using 112 clinical paraffin-embedded specimens. In the current study, while we have not evaluated EMMPRIN expression using pre-operative endometrial materials, the measurement of EMMPRIN may serve as an additional tool for endometrial carcinoma diagnosis including pre-operative evaluation.

MMP expression has been demonstrated to be associated with cancer infiltration and invasion into vessels, suggesting that MMP inhibitors may prolong recurrence-free survival by interfering with tumor infiltration and invasion. In endometrial carcinoma, MMP-7, a member of the MMP family, has been reported to be associated with invasiveness, metastatic spread and poor prognosis (19). Recently, research of EMMPRIN in malignant disease has increased and the expression of EMMPRIN has been reported to correlate with clinical prognosis of patients with several malignancies. It has been reported that expression of EMMPRIN protects cancer cells from anoikis through inhibition of Bim (20). However, the molecular mechanisms underlying the actions of EMMPRIN and relation to MMPs are not fully understood, and no report has demonstrated blockade of EMMPRIN molecules in malignant diseases.

As a future direction, MoAb 12C3 may also be useful as a targeting agent for cancer imaging and/or chemotherapy. Further investigations are necessary to elucidate EMMPRIN's function including its relationship with MMP expression in endometrial carcinoma.

\section{Acknowledgements}

This study was supported by Grant-in-Aid for Scientific Research, Japan (no. 16659455).

\section{References}

1. Tang Y, Nakada MT, Kesavan P, McCabe F, Millar H, Rafferty P, Bugelski P and Yan L: Extracellular matrix metalloproteinase inducer stimulates tumor angiogenesis by elevating vascular endothelial cell growth factor and matrix metalloproteinases. Cancer Res 65: 3193-3199, 2005.

2. Kanekura T, Chen X and Kanzaki T: Basigin (CD147) is expressed on melanoma cells and induces tumor cell invasion by stimulating production of matrix metalloproteinases by fibroblasts. Int J Cancer 99: 520-528, 2002. 
3. Marionnet C, Lalou C, Mollier K, Chazal M, Delestaing G, Compan D, Verola O, Vilmer C, Cuminet J, Dubertret L and Basset-Seguin N: Differential molecular profiling between skin carcinomas reveals four newly reported genes potentially implicated in squamous cell carcinoma development. Oncogene 22: 3500-3505, 2003.

4. Sier CF, Zuidwijk K, Zijlmans HJ, Hanemaaijer R, MulderStapel AA, Prins FA, Dreef EJ, Kenter GG, Fleuren GJ and Gorter A: EMMPRIN-induced MMP-2 activation cascade in human cervical squamous cell carcinoma. Int J Cancer 118: 2991-2998, 2006.

5. Davidson B, Konstantinovsky S, Nielsen S, Dong HP, Berner A, Vyberg M and Reich R: Altered expression of metastasisassociated and regulatory molecules in effusions from breast cancer patients: a novel model for tumor progression. Clin Cancer Res 10: 7335-7346, 2004.

6. Reimers N, Zafrakas K, Assmann V, Egen C, Riethdorf L, Riethdorf S, Berger J, Ebel S, Janicke F, Sauter G and Pantel K: Expression of extracellular matrix metalloproteases inducer on micrometastatic and primary mammary carcinoma cells. Clin Cancer Res 10: 3422-3428, 2004.

7. Davidson B, Goldberg I, Berner A, Kristensen GB and Reich R: EMMPRIN (extracellular matrix metalloproteinase inducer) is a novel marker of poor outcome in serous ovarian carcinoma. Clin Exp Metastasis 20: 161-169, 2003.

8. Ishibashi Y, Matsumoto T, Niwa M, Suzuki Y, Omura N, Hanyu N, Nakada K, Yanaga K, Yamada K, Ohkawa K, Kawakami M and Urashima M: CD147 and matrix metalloproteinase-2 protein expression as significant prognostic factors in esophageal squamous cell carcinoma. Cancer 101: 1994-2000, 2004.

9. Jin JS, Hsieh DS, Lin YF, Wang JY, Sheu LF and Lee WH: Increasing expression of extracellular matrix metalloprotease inducer in renal cell carcinoma: tissue microarray analysis of immunostaining score with clinicopathological parameters. Int J Urol 13: 573-580, 2006.

10. Rosenthal EL, Shreenivas S, Peters GE, Grizzle WE, Desmond R and Gladson CL: Expression of extracellular matrix metalloprotease inducer in laryngeal squamous cell carcinoma. Laryngoscope 113: 1406-1410, 2003.

11. Zheng H, Takahashi H, Murai Y, Cui Z, Nomoto K, Miwa S, Tsuneyama K and Takano Y: Pathobiological characteristics of intestinal- and diffuse-type gastric carcinoma in Japan: an immunostaining study on the tissue microarray. J Clin Pathol (In press).
12. Lax SF, Kurman RJ, Pizer ES, Wu L and Ronnett BM: A binary architectural grading system for uterine endometrial endometrioid carcinoma has superior reproducibility compared with FIGO grading and identifies subsets of advance-stage tumors with favorable and unfavorable prognosis. Am J Surg Pathol 24: 1201-1208, 2000.

13. Lurain JR: Uterine cancer. In: Novak's Gynecology. 13th edition. Berek JS (ed). Lippincott Williams \& Wilkins, Philadelphia, PA, USA, pp1143-1197, 2002.

14. Zaino RJ, Kurman RJ, Diana KL and Morrow CP: The utility of the revised International Federation of Gynecology and Obstetrics histologic grading of endometrial adenocarcinoma using a defined nuclear grading system. A Gynecologic Oncology Group study. Cancer 75: 81-86, 1995.

15. Yamada K, Ohkawa K and Joh K: Monoclonal antibody, Mab $12 \mathrm{C} 3$, is a sensitive immunohistochemical marker of early malignant change in epithelial ovarian tumor. Am J Clin Pathol 103: 288-294, 1995.

16. Morrow CP, Bundy BN, Kurman RJ, Creasman WT, Heller P, Homesley HD and Graham JE: Relationship between surgicalpathological risk factors and outcome in clinical stage I and II carcinoma of the endometrium: a Gynecologic Oncology Group study. Gynecol Oncol 40: 55-65, 1991.

17. Murray SK, Young RH and Scully RE: Unusual epithelial and stromal changes in myoinvasive endometrioid adenocarcinoma: a study of their frequency, associated diagnostic problems, and prognostic significance. Int J Gynecol Pathol 22: 324-333, 2003 .

18. Nordstrom B, Bergstrom R and Strang P: Prognostic index models in stage I and II endometrial carcinoma. Anticancer Res 18: 3717-3724, 1998.

19. Misugi F, Sumi T, Okamoto E, Nobeyama H, Hattori K, Yoshida H, Matsumoto Y, Yasui T, Honda K and Ishiko O: Expression of matrix metalloproteinases and tissue inhibitors of metalloproteinase in uterine endometrial carcinoma and a correlation between expression of matrix metalloproteinase-7 and prognosis. Int J Mol Med 16: 541-546, 2005.

20. Yang JM, O'Neill P, Jin W, Foty R, Medina DJ, Xu Z, Lomas M, Arndt GM, Tang Y, Nakada M, Yan L and Hait WN: Extracellular matrix metalloproteinase inducer (CD147) confers resistance of breast cancer cells to Anoikis through inhibition of Bim. J Biol Chem 281: 9719-9727, 2006. 